\title{
Racial Differences in Age-Related Variations of Testosterone Levels Among US Males: Potential Implications for Prostate Cancer and Personalized Medication
}

\author{
Hui Hu • Folakemi T. Odedina • Romonia R. Reams • \\ Claudia T. K. Lissaker • Xiaohui Xu
}

Received: 2 April 2014 /Revised: 7 August 2014 / Accepted: 22 August 2014 / Published online: 11 September 2014

(C) W. Montague Cobb-NMA Health Institute 2014

\begin{abstract}
Aim The magnitude of the age-related declines in testosterone rather than levels measured at single point in time may be related to the genesis of prostate cancer (PCa). We examined age-related variations of testosterone levels among black and white males, which may provide important insights into racial disparities in PCa incidence and mortality.

Method We analyzed data from the 1999-2004 National Health and Nutritional Examination Survey to compare agerelated variations in the testosterone levels of 355 black and 631 white males.

Result Overall, between the ages of 12 and 15, black males had lower testosterone levels than white males. Testosterone levels increased rapidly with age and reached higher and earlier peak levels in black males compared to white males at 20-30 years of age. After reaching a peak level, testosterone levels declined earlier in blacks than in whites. Further analyses showed that black males had considerably higher levels of testosterone compared to white males aged 20-39 years after adjusting for covariates, including age, body mass index, cigarette smoking, physical activity, and waist circumference; however, no statistically significant differences were observed between the groups at any other age.
\end{abstract}

H. Hu $\cdot$ C. T. K. Lissaker $\cdot$ X. Xu $(\bowtie)$

Department of Epidemiology, College of Public Health and Health

Professions and College of Medicine, University of Florida,

Gainesville, FL, USA

e-mail: xhxu@PHHP.UFL.EDU

F. T. Odedina

Department of Radiation Oncology, College of Medicine, University of Florida, Gainesville, FL, USA

\section{R. R. Reams}

Division of Basic Pharmaceutical Sciences,

College of Pharmacy \& Pharmaceutical Sciences, Florida A\& M

University, Tallahassee, FL, USA
Conclusion Our study revealed that testosterone levels in black males decrease substantially with increasing age compared to those in white males. This rapid drop in testosterone levels may contribute to racial disparities in PCa. Our findings also suggest that personalized medication for hormone replacement therapy may be necessary to avoid sudden drops in testosterone levels, particularly for black males.

Keywords Racial disparity · Testosterone · Prostate cancer . Testosterone change

$\begin{array}{ll}\text { Abbreviations } \\ \text { BMI } & \text { Body mass index } \\ \text { CIs } & \text { Confidence intervals } \\ \text { MET } & \text { Metabolic equivalent scores } \\ \text { NHANES } & \begin{array}{l}\text { National Health and Nutritional Examination } \\ \text { Surveys }\end{array} \\ \text { PCa } & \begin{array}{l}\text { Prostate cancer } \\ \text { MEC }\end{array} \\ \text { ME } & \text { Mobile examination center } \\ \text { LOESS } & \text { Locally weighted scatterplot smoothing }\end{array}$

\section{Introduction}

Disparities in prostate cancer (PCa) incidence and mortality between black and white males have been well documented $[1,2]$. According to the American Cancer Society's Cancer Facts and Figures 2014, black males have the highest PCa incidence $(220$ per 100,000$)$ and mortality $(50.9$ per 100,000$)$ rates, while white males have relatively low incidence (138.6 per 100,000$)$ and mortality $(21.3$ per 100,000$)$ rates [3]. Although some studies have reported no statistically significant 
differences between the circulating levels of testosterone of black and white males [4-9], others have reported higher levels in black males [10-14]. Given these inconsistent results, testosterone levels do not seem to provide a clear answer to the problem of racial disparities in PCa. These studies all share the common characteristic of comparing testosterone levels measured at single point in time by race; however, this may not be the best way to investigate the role of testosterone in PCa racial disparities.

There is no doubt that testosterone plays an important role in the development of PCa. Testosterone is required for the normal development of the prostate, which will atrophy if androgen support is withdrawn [15]. Prevailing wisdom indicates that high-testosterone levels in aging males could stimulate prostatic tissue growth and provide an increased opportunity for mutation and carcinogenesis, as shown in experimental studies that androgen administration can produce and/ or accelerate prostate cancer $[16,17]$. Moreover, the progression of prostate cancer is often dependent upon testosterone, and some studies have shown that this cancer regresses if the patient is castrated $[18,19]$. However, others have failed to detect this correlation [20] and even reported an inverse association between testosterone and PCa [21-24]. Thus, inconsistent findings in both areas suggest that a fresh perspective on the relationship between testosterone and $\mathrm{PCa}$ as well as its role in racial disparities is in order.

As men grow older, their androgen levels decrease and their PCa risk increases. The age-related patterns of $\mathrm{PCa}$ and testosterone suggest that the magnitude of the age-related declines in testosterone may play a critical role in prostatic carcinogenesis. With aging, testosterone levels fall below a particular threshold, which in turn may trigger the onset of PCa. This threshold could be different for individuals (i.e., individualized threshold level) since it may be related to an individual's baseline or peak level of testosterone at a young age. Previous studies compared testosterone levels measured at a single point in time rather than the magnitude of the agerelated decline in testosterone between cases and controls or among race groups, which may account for their inconsistent findings.

As longitudinal data regarding lifetime testosterone levels are limited, a cross-sectional examination of age-related patterns of testosterone levels by race may provide important insights into disparities in testosterone changes and contribute to our understanding of the role of testosterone in the development of PCa. In this study, we analyzed data from the 1999-2004 National Health and Nutritional Examination Surveys (NHANES) to examine variations in the degree of serum testosterone concentrations in black and white males aged $\geq 12$ years old. We also examined the potential implications of these variations in $\mathrm{PCa}$ racial disparities.

\section{Materials and Methods}

Study Population

The NHANES is a population-based survey designed to collect information on the health and nutrition of US households and to obtain a representative sample of the non-institutional civilian US population [25]. Because of the availability of testosterone concentrations measured in 2-year cycles, we analyzed data from the 1999-2004 NHANES (3 cycles) to test the hypothesis that variations in age-related testosterone levels differ between black and white males. Testosterone levels were measured in a subsample of 1,554 participants aged 12 years and older in the 1999-2004 NHANES data. Participants previously diagnosed with $\mathrm{PCa}$ were excluded $(n=18)$. Participants were also excluded if their race/ethnicity was anything other than white or black $(n=550)$. Most notably, the proportion of non-Mexican-American Hispanic respondents in the 1999-2004 NHANES was smaller than in the US population, leading to unstable estimates for all Hispanic males [26]. A total of 986 participants met the above criteria and were analyzed in this study.

\section{Measurement of Serum Testosterone Levels}

We obtained total, free, and bioavailable testosterone data for our selected cohorts. The CDC provided a standard protocol for testosterone measurements [27]. Briefly, the original whole blood specimens were collected during physical examinations in a mobile examination center (MEC), processed, frozen, and shipped to the National Center for Environmental Health for testing. Concentrations of total testosterone were assessed in stored serum specimens from men selected for organochlorine pesticide measurements in the 1999-2004 NHANES. Serum concentrations of total testosterone were measured by competitive electrochemiluminescence immunoassay. The lowest detection limit for testosterone was $0.02 \mathrm{ng} / \mathrm{mL}$. Mass action equations were used to estimate free and bioavailable testosterone levels based on concentrations from measured testosterone, sex hormonebinding globulin, and albumin [28].

\section{Covariates}

All covariates were carefully selected based on previous studies [4-14]. Age (12-19, 20-29, 30-39, 40-49, 50-59, 60-69, or $70+$ ) was obtained from a demographic questionnaire. Physical measurements, including body mass index (BMI; $<18.5,18.5-25.0,25.0-30.0$, or $\geq 30.0$ ) and waist circumference (categorized into tertiles), measured during the physical examination in the MEC were also obtained. In addition, cigarette smoking was assessed by serum cotinine concentrations and self-reported questionnaire. Participants with a 
serum cotinine $\geq 15 \mathrm{ng} / \mathrm{mL}$ were categorized as current smokers regardless of the self-reported data, and those with serum cotinine $<15 \mathrm{ng} / \mathrm{mL}$ were categorized into three groups (i.e., current smoker, former smoker, or non-smoker) based on self-reported data. Leisure-time physical activity was assessed by metabolic equivalent (MET) scores per 30 days calculated based on answers to the questionnaire. Participants with MET scores equal to 0 were categorized as sedentary, while others were categorized into low-activity and high-activity groups based on the median.

\section{Statistical Analyses}

Descriptive statistics, such as Wald chi-square analysis, was performed when appropriate. Total, free, and bioavailable testosterone levels were log-transformed to account for the skewed distribution. The Student's $t$ test was used to compare the geometric means of testosterone by race and age. We also fitted locally weighted scatterplot smoothing (LOESS) curves of testosterone with $95 \%$ confidence limits by race and age. In addition, linear regression models were used to assess the relationship between race/ethnicity and testosterone in stratified age groups (i.e., $12-19,20-39,40-59$, and $\geq 60$ years). The fully adjusted model included covariates, such as age, BMI, smoking, physical activity, and waist circumference. We hypothesized that black males may have higher degrees of lifetime changes in testosterone levels compared to white males. Thus, using multivariable linear regression models with white males as the reference group, we calculated $\beta$ coefficients and $95 \%$ confidence intervals (CIs) for testosterone levels in association with race/ethnicity for each stratified age group. The sample weights, stratification, and clustering design variables were incorporated into all SAS survey procedures to ensure the correct estimation of sampling error. A 6-year subsample weight was calculated for the combined 1999-2004 data by following the NHANES analytic and reporting guidelines. This calculated weight was used to analyze the combined 6-year data for the 1999-2004 NHANES. The standard error (SE) was estimated using the Taylor series linearization method [29]. All statistical analyses were performed using SAS 9.3 software (SAS Institute Inc., Cary, NC) survey procedures.

\section{Results}

This study analyzed data from 986 PCa-free participants from the 1999-2004 NHANES. Table 1 presents the distribution of selected demographic characteristics and covariates by race. White males were older $(P<0.01)$ and had higher BMI $(P=0.04)$ and waist circumferences $(P<0.01)$, whereas black males were more likely to be current smokers and sedentary
$(P<0.01)$. Figure 1 illustrates the fitted LOESS curves of testosterone levels by race and age. At the ages of $12-15$ years, black males had lower levels of testosterone than white males. Testosterone levels increased rapidly with increasing age and reached higher and earlier peak levels in black males than in white males. After reaching a peak level, testosterone declined at earlier ages in black males.

Table 2 shows the geometric mean serum concentrations of testosterone by race and age. Black males had higher concentrations of total, free, and bioavailable testosterone compared to white males. When stratified by age, black males had significantly higher total $(P<0.01)$ and free testosterone $(P<0.01)$ compared to white males aged $20-39$ years. However, statistically significant differences were not observed in any other age groups.

Table 3 shows the associations between race and testosterone levels after adjusting for age and other covariates, including BMI, cigarette smoking, physical activity, and waist circumference. Black males had higher total testosterone levels than white males ( $\beta$ coefficient, $0.07 ; 95 \% \mathrm{CI}, 0.00-0.13$ ). When stratified by age, this difference was only observed among males aged $20-39$ years ( $\beta$ coefficient, $0.12 ; 95 \%$ CI, 0.04-0.21). No statistically significant differences were observed in any other age groups.

\section{Discussion}

Using a large nationally representative sample from the NHANES, we found that black and white males had different age-related patterns of testosterone. These results suggest that the magnitude of age-related declines in testosterone differs between black and white males. Notably, these findings may have implications for PCa racial disparities. As we described previously, each individual may have his own threshold of testosterone for prostatic carcinogenesis, which may be associated with his baseline or peak testosterone levels. If the baseline testosterone level is high, the threshold may be relatively higher as the normal prostate is acclimated to a hightestosterone level. If this is true, the relatively higher testosterone levels among young adult black males (20-30 years old) may indicate that they also have relatively higher thresholds compared to white males. We also found that black males experienced a more dramatic age-related change in testosterone levels than white males. These results suggest that testosterone levels more frequently fall below the necessary threshold in black males, which may eventually lead to the development of PCa and help explain racial disparities in PCa. However, this hypothesis warrants further investigation.

In addition, the magnitude of age-related testosterone declines could also help explain inconsistent findings regarding the relationship between testosterone and PCa. The prevailing wisdom that high-testosterone levels are a risk factor for $\mathrm{PCa}$ 
Table 1 Characteristics of 986 males (NHANES 1999-2004)

\begin{tabular}{|c|c|c|c|c|c|}
\hline & \multicolumn{2}{|c|}{ Whites $(n=631)$} & \multicolumn{2}{|c|}{ Blacks $(\mathrm{n}=355)$} & \multirow[t]{2}{*}{$p$ value } \\
\hline & $n$ & Percent $^{\mathrm{a}}(95 \% \mathrm{CI})$ & $n$ & Percent $^{\mathrm{a}}(95 \% \mathrm{CI})$ & \\
\hline Age $(\%)$ & & & & & $<0.01$ \\
\hline 12-19 years & 138 & $12.8(10.8-14.7)$ & 177 & $19.8(16.6-23.0)$ & \\
\hline 20-39 years & 148 & $32.0(27.0-37.0)$ & 80 & $41.2(35.5-46.9)$ & \\
\hline $40-59$ years & 163 & $36.6(32.1-41.1)$ & 56 & $28.5(23.1-33.9)$ & \\
\hline$\geq 60$ years & 182 & $18.6(16.0-21.3)$ & 42 & $10.5(7.0-14.1)$ & \\
\hline BMI (\%) & & & & & 0.04 \\
\hline$<18.5$ & 29 & $3.0(1.8-4.2)$ & 29 & $5.2(3.0-7.4)$ & \\
\hline $18.5-25.0$ & 220 & $30.8(27.1-34.6)$ & 164 & $40.1(34.0-46.1)$ & \\
\hline $25.0-30.0$ & 223 & $38.6(34.2-42.9)$ & 85 & $29.5(24.8-34.3)$ & \\
\hline$\geq 30.0$ & 158 & $27.6(23.5-31.7)$ & 76 & $25.2(20.9-29.5)$ & \\
\hline Smoking (\%) & & & & & $<0.01$ \\
\hline Current smoker & 195 & $33.4(29.1-37.7)$ & 128 & $40.8(32.8-48.8)$ & \\
\hline Former smoker & 189 & $26.7(22.7-30.6)$ & 61 & $14.0(10.1-17.9)$ & \\
\hline Non-smoker & 247 & $40.0(34.8-45.1)$ & 166 & $45.2(37.7-52.6)$ & \\
\hline Physical activity (\%) & & & & & 0.01 \\
\hline Sedentary & 190 & $28.7(25.0-32.3)$ & 110 & $37.0(31.0-43.0)$ & \\
\hline Low & 253 & $42.8(39.0-46.6)$ & 95 & $30.1(23.0-37.1)$ & \\
\hline High & 185 & $28.5(25.0-32.0)$ & 141 & $32.9(27.4-38.5)$ & \\
\hline Waist circumference $(\%)$ & & & & & $<0.01$ \\
\hline Low & 143 & $18.1(14.5-21.8)$ & 177 & $38.9(33.2-44.6)$ & \\
\hline Medium & 224 & $39.3(35.4-43.3)$ & 97 & $35.7(29.9-41.6)$ & \\
\hline High & 248 & $42.5(38.3-46.8)$ & 73 & $25.4(20.6-30.1)$ & \\
\hline
\end{tabular}

${ }^{\mathrm{a}}$ Weighted percent with $95 \%$ confidence interval

is not supported by extensive evidence [23]. In fact, recent studies refute this notion. First, it is inconsistent with the agerelated pattern of PCa risk. Although males exhibit their peak levels of testosterone between the ages of 20 and 30 years, this is not the highest risk period for the development of $\mathrm{PCa}$; indeed, approximately $90 \%$ of PCa cases are diagnosed after the age of 60 years old when testosterone levels have already declined to relatively low levels. Moreover, recent studies have shown that low testosterone is associated with an increased risk of PCa on biopsy, high Gleason scores, seminal vesicle invasion, and biochemical recurrence [24]. Thus, comparing testosterone levels measured at a single point in time, as most previous studies have done, is not adequate for an investigation into the relationship between testosterone and PCa. We theorize that PCa patients can have relatively higher or lower testosterone at the time of diagnosis, as levels at a single point in time are irrelevant to the genesis of $\mathrm{PCa}$; however, the magnitude of age-related declines in testosterone levels is significant. These assumptions allow us to explain the inconsistent findings regarding the relationship between testosterone and PCa. For example, men who have high levels of testosterone at young ages may have levels that remain relatively high at the time of PCa diagnosis even after a significant fall in testosterone with aging. On the other hand, men who exhibit low levels of testosterone at a young age may have even lower testosterone levels at the time of PCa diagnosis. If a study includes both groups, then it is plausible that testosterone levels at diagnosis will not be associated with $\mathrm{PCa}$; this helps to explain the null associations found in a recent collaborative analysis of 18 prospective studies [20]. Therefore, the inconsistent findings from previous studies may be attributable to the focus on testosterone levels measured at a single point in time as opposed to the magnitude of age-related declines in testosterone [18-24, 30, 31].

As we used cross-sectional data for this study, we were not able to investigate the difference in lifetime testosterone variations between PCa cases and controls. However, recent studies in PCa patients have found that lower testosterone is associated with advanced pathologic stage, high Gleason score, and biochemical recurrence after radical prostatectomy [32]. If it is true that men diagnosed with $\mathrm{PCa}$ and controls have similar levels of testosterone and/or that men who eventually develop PCa have higher testosterone levels at a young age, then it is also likely that men with $\mathrm{PCa}$ experience more significant declines in testosterone throughout a lifetime. Furthermore, studies have reported higher PCa prevalence in 

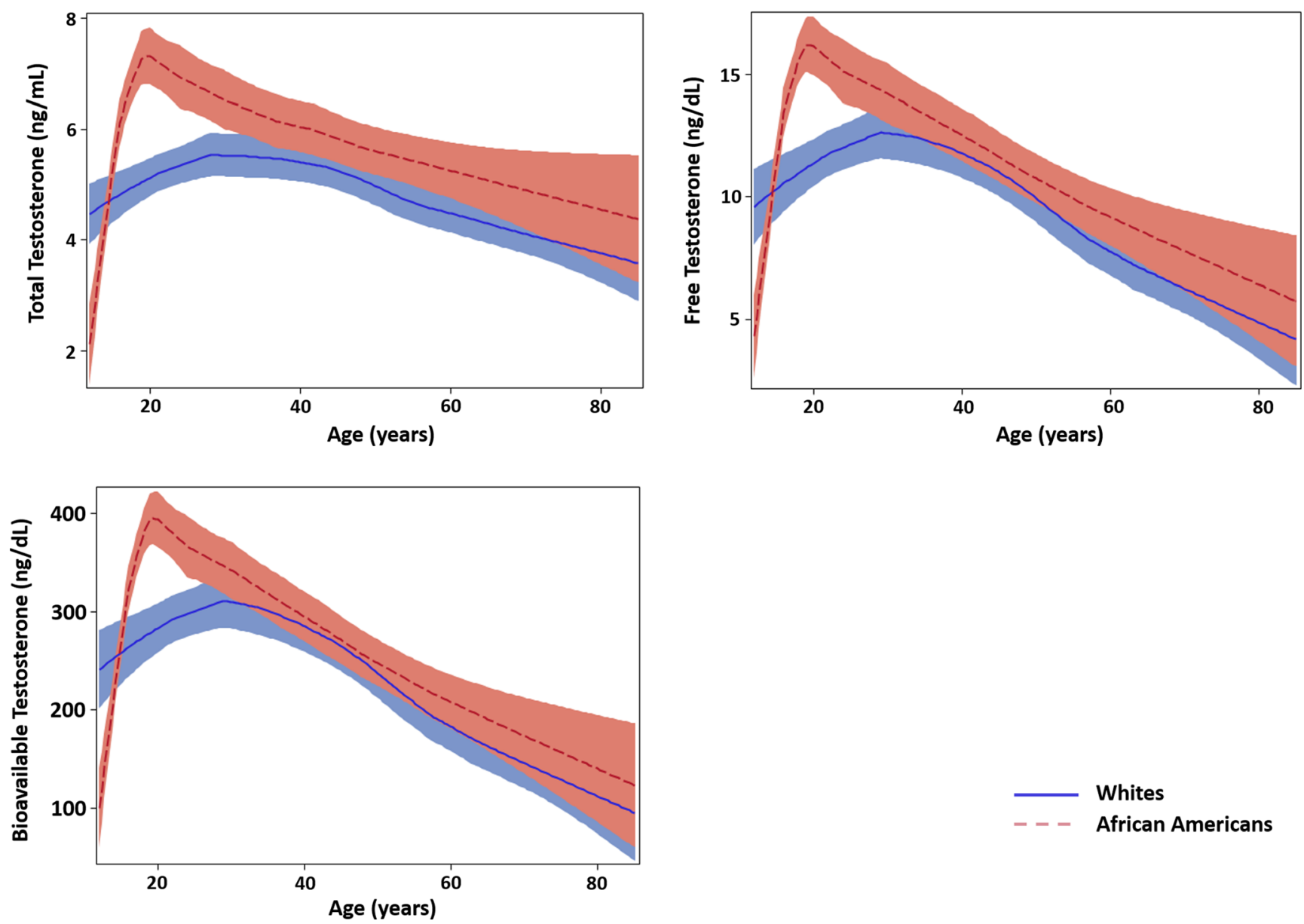

Whites

-- African Americans

Fig. 1 Fitted LOESS curves with $95 \%$ confidence limits of unadjusted testosterone by race and age

hypogonadal men [21, 23], further supporting the hypothesis that a significant decline in testosterone may be related to the development of PCa. These findings may have implications for hormone replacement therapy as well. Due to individual
Table 2 Geometric mean (95\% confidence interval) serum concentrations of sex steroid hormones by race in 986 males (NHANES 1999-2004)

\begin{tabular}{llll}
\hline & Whites $(n=631)$ & Blacks $(n=355)$ & $p$ value \\
\hline Total testosterone $(\mathrm{ng} / \mathrm{mL})$ & $4.27(4.08-4.47)$ & $5.15(4.81-5.51)$ & $<0.01$ \\
12-19 years & $3.35(2.67-4.20)$ & $3.60(2.92-4.44)$ & 0.65 \\
20-39 years & $5.04(4.71-5.41)$ & $6.29(5.72-6.91)$ & $<0.01$ \\
40-59 years & $4.39(4.02-4.78)$ & $5.24(4.65-5.92)$ & 0.12 \\
$\geq 60$ years & $3.59(3.26-3.95)$ & $4.32(3.74-4.99)$ & 0.13 \\
Free testosterone $(\mathrm{ng} / \mathrm{dL})$ & $8.44(7.94-8.96)$ & $10.24(9.46-11.08)$ & $<0.01$ \\
$12-19$ years & $6.31(4.76-8.37)$ & $7.09(5.53-9.07)$ & 0.55 \\
20-39 years & $11.50(10.95-12.08)$ & $13.56(12.27-14.98)$ & $<0.01$ \\
$40-59$ years & $8.80(7.95-9.73)$ & $10.16(8.97-11.52)$ & 0.64 \\
$\geq 60$ years & $5.50(4.99-6.07)$ & $6.84(6.05-7.72)$ & 0.07 \\
Bioavailable testosterone $(\mathrm{ng} / \mathrm{dL})$ & $202.79(190.90-215.41)$ & $240.24(221.60-260.44)$ & $<0.01$ \\
$12-19$ years & $157.39(118.42-209.19)$ & $170.15(132.57-218.38)$ & 0.69 \\
$20-39$ years & $281.23(266.34-296.95)$ & $327.18(294.97-362.91)$ & 0.06 \\
$40-59$ years & $209.38(189.43-231.43)$ & $231.36(203.48-263.06)$ & 0.90 \\
$\geq 60$ years & $127.52(115.49-140.81)$ & $149.44(131.13-170.31)$ & 0.23 \\
\hline
\end{tabular}


Table 3 Adjusted regression models of the associations between race and log-transformed testosterone in 986 males (NHANES 1999-2004)

\begin{tabular}{|c|c|c|c|c|c|c|}
\hline & \multicolumn{2}{|l|}{ Total testosterone } & \multicolumn{2}{|l|}{ Free testosterone } & \multicolumn{2}{|c|}{ Bioavailable testosterone } \\
\hline & Estimate $(95 \% \mathrm{CI})$ & $p$ value & Estimate $(95 \% \mathrm{CI})$ & $p$ value & Estimate $(95 \% \mathrm{CI})$ & $p$ value \\
\hline \multicolumn{7}{|l|}{ Age-adjusted $^{\mathrm{a}}$} \\
\hline \multicolumn{7}{|l|}{ Overall } \\
\hline Whites & Ref. & & Ref. & & Ref. & \\
\hline Blacks & $0.16(0.08,0.24)$ & $<0.01$ & $0.12(0.04,0.21)$ & $<0.01$ & $0.09(0.01,0.17)$ & 0.04 \\
\hline \multicolumn{7}{|c|}{$12-19$ years } \\
\hline Whites & Ref. & & Ref. & & Ref. & \\
\hline Blacks & $0.04(-0.22,0.30)$ & 0.75 & $0.10(-0.21,0.41)$ & 0.53 & $0.06(-0.25,0.37)$ & 0.71 \\
\hline \multicolumn{7}{|c|}{ 20-39 years } \\
\hline Whites & Ref. & & Ref. & & Ref. & \\
\hline Blacks & $0.22(0.09,0.35)$ & $<0.01$ & $0.16(0.05,0.28)$ & $<0.01$ & $0.15(0.03,0.27)$ & 0.02 \\
\hline \multicolumn{7}{|c|}{$40-59$ years } \\
\hline Whites & Ref. & & Ref. & & Ref. & \\
\hline Blacks & $0.14(0.00,0.28)$ & 0.05 & $0.07(-0.08,0.21)$ & 0.35 & $0.02(-0.13,0.17)$ & 0.77 \\
\hline \multicolumn{7}{|l|}{$\geq 60$ years } \\
\hline Whites & Ref. & & Ref. & & Ref. & \\
\hline Blacks & $0.17(0.00,0.33)$ & 0.04 & $0.17(0.03,0.32)$ & $<0.01$ & $0.11(-0.05,0.28)$ & 0.16 \\
\hline \multicolumn{7}{|c|}{ Fully adjusted $^{\mathrm{b}}$} \\
\hline \multicolumn{7}{|c|}{ Overall } \\
\hline Whites & Ref. & & Ref. & & Ref. & \\
\hline Blacks & $0.07(0.00,0.13)$ & 0.05 & $0.06(-0.02,0.15)$ & 0.13 & $0.03(-0.06,0.11)$ & 0.54 \\
\hline \multicolumn{7}{|c|}{$12-19$ years } \\
\hline Whites & Ref. & & Ref. & & Ref. & \\
\hline Blacks & $-0.02(-0.27,0.24)$ & 0.90 & $0.02(-0.30,0.34)$ & 0.90 & $-0.02(-0.34,0.30)$ & 0.90 \\
\hline \multicolumn{7}{|c|}{ 20-39 years } \\
\hline Whites & Ref. & & Ref. & & Ref. & \\
\hline Blacks & $0.12(0.04-0.21)$ & $<0.01$ & $0.09(-0.01,0.19)$ & 0.08 & $0.07(-0.04,0.17)$ & 0.20 \\
\hline \multicolumn{7}{|c|}{$40-59$ years } \\
\hline Whites & Ref. & & Ref. & & Ref. & \\
\hline Blacks & $0.01(-0.14,0.16)$ & 0.91 & $-0.03(-0.18,0.13)$ & 0.73 & $-0.08(-0.23,0.07)$ & 0.28 \\
\hline \multicolumn{7}{|l|}{$\geq 60$ years } \\
\hline Whites & Ref. & & Ref. & & Ref. & \\
\hline Blacks & $0.10(-0.06,0.26)$ & 0.21 & $0.13(-0.01,0.27)$ & 0.06 & $0.08(-0.08,0.24)$ & 0.31 \\
\hline
\end{tabular}

${ }^{\text {a }}$ Adjusted for age

${ }^{\mathrm{b}}$ Adjusted for age, BMI, cigarette smoking, physical activity, and waist circumference

hormonal variations, patients may require different therapeutic amounts. Personalized medication may improve the outcome of hormone replacement therapy.

Several aspects of our study merit further discussion. First, the NHANES data provided us with a unique opportunity to study racial disparities in age-related variations of testosterone in a large nationally representative sample. However, given the cross-sectional design of the NHANES, we cannot assess differences in an individual's hormonal trajectory over a lifetime by race. Trends in testosterone levels may be affected by potential period or cohort effects; moreover, the trend in racial differences might also be partially explained by temporal changes. Although we were able to adjust for many potential confounders in this study, residual confounders (e.g., puberty status, which was not available in the data) may be possible. Finally, as our data were drawn from participants free of $\mathrm{PCa}$, we could not directly investigate the magnitude of testosterone decline among patients with PCa or whether different hormonal profiles and trajectories over a lifetime resulted in PCa. Future studies will include a cohort design and long-term follow-up to further examine this hypothesis. 


\section{Conclusion}

In a large nationally representative sample of US adults, we found racial disparities in age-related variations of testosterone levels between black and white males. Our results indicate that black males may experience more significant testosterone changes throughout their lifetimes compared to white males. The magnitude of age-related declines in testosterone may be implicated in PCa incidence and mortality disparities between black and white males. These findings warrant further investigation in a prospective study.

Acknowledgments The authors would like to thank Katherine Ann Casey-Sawicki, MA, in the Department of Radiation Oncology at the University of Florida for her significant editorial contributions to this paper.

Conflict of Interest Hui Hu, Folakemi T. Odedina, Romania R. Reams, Claudia T K Lissaker, and Xiaohui Xu declare that they have no conflict of interest.

Ethical Statement All procedures followed were in accordance with the ethical standards of the responsible committee on human experimentation (institutional and national) and with the Helsinki Declaration of 1975 , as revised in 2000. Informed consent was obtained from all subjects for being included in the study by NHANES.

\section{References}

1. Krieg M, Nass R, Tunn S. Effect of aging on endogenous level of 5 alpha-dihydrotestosterone, testosterone, estradiol, and estrone in epithelium and stroma of normal and hyperplastic human prostate. J Clin Endocrinol Metab. 1993;77(2): 375-81.

2. Walsh PC. Screening for prostate cancer: an update of the evidence for the U.S. Preventive Services Task Force. J Urol. 2003;170(1): 312-3.

3. American Cancer Society. Cancer facts and figures 2014. Atlanta: American Cancer Society; 2014.

4. Rohrmann S, Nelson WG, Rifai N, Brown TR, Dobs A, Kanarek N, et al. Serum estrogen, but not testosterone, levels differ between black and white men in a nationally representative sample of Americans. J Clin Endocrinol Metab. 2007;92 (7):2519-25.

5. Wu AH, Whittemore AS, Kolonel LN, John EM, Gallagher RP, West DW, et al. Serum androgens and sex hormone-binding globulins in relation to lifestyle factors in older AfricanAmerican, white, and Asian men in the United States and Canada. Cancer Epidemiol Biomark Prev: Publ Am Assoc Cancer Res cosponsored by the Am Soc Prev Oncol. 1995;4 (7):735-41.

6. Wright NM, Renault J, Willi S, Veldhuis JD, Pandey JP, Gordon L, et al. Greater secretion of growth hormone in black than in white men: possible factor in greater bone mineral density - a clinical research center study. J Clin Endocrinol Metab. 1995;80(8):2291-7.

7. Ettinger B, Sidney S, Cummings SR, Libanati C, Bikle DD, Tekawa IS, et al. Racial differences in bone density between young adult black and white subjects persist after adjustment for anthropometric, lifestyle, and biochemical differences. J Clin Endocrinol Metab. 1997:82(2):429-34.

8. Cheng I, Yu MC, Koh WP, Pike MC, Kolonel LN, Henderson BE, et al. Comparison of prostate-specific antigen and hormone levels among men in Singapore and the United States. Cancer Epidemiol Biomark Prev: Publ Am Assoc Cancer Res cosponsored by the Am Soc Prev Oncol. 2005;14(7):1692-6.

9. Litman HJ, Bhasin S, Link CL, Araujo AB, McKinlay JB. Serum androgen levels in black, Hispanic, and white men. J Clin Endocrinol Metab. 2006;91(11):4326-34.

10. Ellis L, Nyborg H. Racial/ethnic variations in male testosterone levels: a probable contributor to group differences in health. Steroids. 1992;57(2):72-5.

11. Orwoll ES, Nielson CM, Labrie F, Barrett-Connor E, Cauley JA, Cummings SR, et al. Evidence for geographical and racial variation in serum sex steroid levels in older men. J Clin Endocrinol Metab. 2010;95(10):E151-60.

12. Ross R, Bernstein L, Judd H, Hanisch R, Pike M, Henderson B. Serum testosterone levels in healthy young black and white men. J Natl Cancer Inst. 1986;76(1):45-8.

13. Winters SJ, Brufsky A, Weissfeld J, Trump DL, Dyky MA, Hadeed V. Testosterone, sex hormone-binding globulin, and body composition in young adult African American and Caucasian men. Metab Clin Exp. 2001;50(10):1242-7.

14. Gapstur SM, Gann PH, Kopp P, Colangelo L, Longcope C, Liu K. Serum androgen concentrations in young men: a longitudinal analysis of associations with age, obesity, and race. The CARDIA male hormone study. Cancer Epidemiol Biomark Prev. 2002;11(10): 1041-7.

15. Imamoto T, Suzuki H, Yano M, Kawamura K, Kamiya N, Araki K, et al. The role of testosterone in the pathogenesis of prostate cancer. Int J Urol. 2008;15(6):472-80.

16. Noble RL. The development of prostatic adenocarcinoma in $\mathrm{Nb}$ rats following prolonged sex hormone administration. Cancer Res. 1977;37(6):1929-33.

17. Pollard M. Lobund-Wistar rat model of prostate cancer in man. Prostate. 1998;37(1):1-4.

18. Huggins C, Hodges CV. Studies on prostatic cancer: I. The effect of castration, of estrogen and of androgen injection on serum phosphatases in metastatic carcinoma of the prostate. CA Cancer J Clin. 1972;22(4):232-40.

19. Huggins C, Stevens R, Hodges CV. Studies on prostatic cancer II. The effects of castration on advanced carcinoma of the prostate gland. Arch Surg. 1941;43(2):209-23.

20. Roddam AW, Allen NE, Appleby P, Key TJ. Endogenous sex hormones and prostate cancer: a collaborative analysis of 18 prospective studies. J Natl Cancer Inst. 2008;100(3):170-83.

21. Morgentaler A, Bruning 3rd CO, DeWolf WC. Occult prostate cancer in men with low serum testosterone levels. JAMA :J Am Med Assoc. 1996;276(23):1904-6.

22. Morgentaler A, Rhoden EL. Prevalence of prostate cancer among hypogonadal men with prostate-specific antigen levels of $4.0 \mathrm{ng} / \mathrm{mL}$ or less. Urology. 2006;68(6):1263-7.

23. Morgentaler A. Testosterone and prostate cancer: an historical perspective on a modern myth. Eur Urol. 2006;50(5):935-9.

24. Morgentaler A. Turning conventional wisdom upside-down: low serum testosterone and high-risk prostate cancer. Cancer. 2011;117(17):3885-8.

25. CDC. Questionnaires, datasets and related documentation: CDC; 2013 [2012.9]. http://www.cdc.gov/nchs/nhanes.htm. Accessed 15 Nov 2013.

26. CDC. National health and nutrition examination survey: analytic guidelines, 1999-2010. Atlanta: CDC, 2013 September, 2013. Report No

27. CDC. Racial/ethnic variation in sex steroid hormone concentrations across age in US men (Surplus Sera) 2013 [2014.6.17]. 
Available from: http://wwwn.cdc.gov/nchs/nhanes/2003-2004/ SSCHL_C.htm. Accessed 15 Nov 2013.

28. Vermeulen A, Verdonck L, Kaufman JM. A critical evaluation of simple methods for the estimation of free testosterone in serum. J Clin Endocrinol Metabol. 1999;84(10):3666-72.

29. Williams RL. Taylor Series Linearization (TSL). Encyclopedia of survey research methods. SAGE Publications, Inc. Thousand Oaks, CA, $877-8$ p.
30. Hsing AW. Hormones and prostate cancer: what's next? Epidemiol Rev. 2001;23(1):42-58.

31. Platz EA, Giovannucci E. The epidemiology of sex steroid hormones and their signaling and metabolic pathways in the etiology of prostate cancer. J Steroid Biochem Mol Biol. 2004;92(4):237-53.

32. Isbarn H, Pinthus JH, Marks LS, Montorsi F, Morales A, Morgentaler A, et al. Testosterone and prostate cancer: revisiting old paradigms. Eur Urol. 2009;56(1):48-56. 